\title{
Postoperative brachytherapy and electron beam irradiation for keloids: A single institution retrospective analysis
}

\author{
QIWEN DUAN ${ }^{1}$, JUNHUA LIU $^{2}$, ZHIGUO LUO $^{1}$ and CHENHAO HU ${ }^{1}$ \\ ${ }^{1}$ Department of Clinical Oncology; ${ }^{2}$ Department of Paediatrics and Epidemiology, \\ Taihe Hospital, Hubei University of Medicine, Shiyan, Hubei 442000, P.R. China
}

Received June 14, 2014; Accepted January 14, 2015

DOI: $10.3892 /$ mco.2015.498

\begin{abstract}
The aim of the present study was to perform a retrospective analysis of the control rate and toxicity of postoperative brachytherapy and electron beam irradiation for keloids. A retrospective review was performed of 116 keloid patients who underwent postoperative brachytherapy and electron beam irradiation between January 1, 2002 and June 30, 2012. Several different radiotherapy techniques and fractionation schedules were performed in the analysis, including high-dose rate (HDR) irradiation with ${ }^{192} \mathrm{Ir}$ at $8 \mathrm{~Gy} / 1$ fraction $(\mathrm{F})+9 \mathrm{~Gy} / 3 \mathrm{~F}$ or $20 \mathrm{~Gy} / 4 \mathrm{~F}$; HDR brachytherapy with ${ }^{60} \mathrm{Co}$ at $20 \mathrm{~Gy} / 4 \mathrm{~F}$ or $18 \mathrm{~Gy} / 6 \mathrm{~F}$; or external beam electron therapy at $26 \mathrm{~Gy} / 13 \mathrm{~F}$ or $30 \mathrm{~Gy} / 15 \mathrm{~F}$. The endpoints of the study were analysis of the control rate and toxicity. The median observation period was 46.5 months (range, 10.0-120.0 months) for all patients. In total, 18 of the 116 patients relapsed, and 16.7 months (range, 10.0-30.0 months) was the median time to recurrence for these patients. The control rates for the patients who received hypofractionation ( $>2$ Gy per fraction) and conventional fraction (2 Gy per fraction) were 88.5 and $76.3 \%$, respectively $(\mathrm{P}=0.043)$. The control rates for the patients whose calculated biological effective doses (BED) were $>30$ Gy and $<30$ Gy were 89.7 and $79.3 \%$, respectively $(\mathrm{P}=0.104)$. There were no grade 2 or higher adverse effects based on the Common Terminology Criteria for Adverse Events v3.0 in the late phase. No evidence was identified for a link between radiotherapy and the subsequent occurrence of cancer. The results of the present study indicate that hypofractionated radiotherapy played an important role as an adjuvant therapy following surgical excision of keloids. A BED of $>30$ Gy appears to be sufficient. No definitive evidence was found for an association between radiotherapy and the occurrence of cancer during the follow-up, however, more cases and longer follow-up periods are required.
\end{abstract}

Correspondence to: Dr Zhiguo Luo, Department of Clinical Oncology, Taihe Hospital, Hubei University of Medicine, 30 South People's Street, Shiyan, Hubei 442000, P.R. China

E-mail:wzfc77@163.com

Key words: brachytherapy, electron beam irradiation, keloid, dose fraction, postoperative irradiation

\section{Introduction}

Keloids are characterized by excessive deposition of collagen in the dermis beyond the boundaries of a wound and they often grow beyond the extent of a cutaneous injury, such as an area of inflammation, a burn or a surgical incision. Spontaneous keloids sometimes develop without a preceding trauma. Although benign, keloids are often aesthetically unpleasant, painful and/or pruritic. Keloids may also be asymptomatic (1). Surgical resection of the keloids alone has been shown to result in a recurrence in 50-80\% of cases (2). Adjuvant treatments, such as radiation therapy, have been utilized to reduce the rate of keloid formation (3). Post-operative radiation therapy is one of the valuable treatment modalities, which substantially reduces the keloid formation (4).

External beam radiation therapy and brachytherapy have been used in the post-operative radiation therapy of keloids. However, to the best of our knowledge, no consensus has been reached on the total dosage, fractionation or optimal timing of the delivery of radiotherapy. The purpose of the present study was to evaluate the efficacy and toxicity of radiotherapy (brachytherapy and electron beam irradiation) following keloid surgery.

\section{Materials and methods}

Patient characteristics. Subsequent to receiving the approval of the Research Ethics Board, the tumor database and radiotherapy records of the Department of Oncology, Taihe Hospital (Shiyan, China) were retrospectively searched and reviewed. Patients were selected with the following characteristics: 15-60 years, Eastern Cooperative Oncology Group performance status of 0 to 2, pathologically confirmed keloids, and treated with surgical excision and radiotherapy. Of the 431 patients, 116 patients were enrolled at the Department of Clinical Oncology, Taihe Hospital, Hubei University of Medicine between January 1, 2002 and June 30, 2012. The characteristics of the 116 patients recruited for the retrospective study are summarized in Table I.

Radiotherapy. Due to the changes in radiation equipment during the past ten years, different radiation techniques were used in the treatment for keloids. Between January 1, 2002 and July 19, 2005, high-dose rate (HDR) brachytherapy 
using Iridium-192 was performed in our center; between August 1,2005 and September 2009, HDR brachytherapy using Cobalt-60 was performed; and between October 1, 2009 and June 30, 2012, a linear accelerator (operating at 4, 6 or $9 \mathrm{Mev}$ ) was used in postoperative radiotherapy of keloids.

For the analyses, the patients treated with each different radiotherapy technique were identified, and the dose and fractionation regimen used for each patient were examined. The patients were subsequently grouped by radiation type and also by dose and fractionation pattern. Only the groups that included $>15$ patients were included in the analyses. Data on the patients in the different groups are shown in detail in Table I.

Of the 116 patients, 44 had received ${ }^{192}$ Ir HDR brachytherapy, 34 had received ${ }^{60} \mathrm{Co}$ HDR brachytherapy and 38 had been treated with electron beam external irradiation. Between January 1, 2002 and July 19, 2005, when HDR ${ }^{192} \mathrm{Ir}$ brachytherapy was performed, all the 44 patients enrolled were administered their first dose of radiation within $12 \mathrm{~h}$ after surgery. Of these 44 patients who had received ${ }^{192}$ Ir HDR brachytherapy, 22 (19\% of the total patients) were treated with a dose of 8 Gy delivered in 10 fractions and 9 Gy delivered in three fractions and $22(19 \%)$ were treated with a dose of 20 Gy delivered in four fractions. Between October 1, 2009 and June 30, 2012, when HDR Cobalt-60 brachytherapy was used in our center as a replacement for ${ }^{192} \mathrm{Ir}$ in the treatment of keloids, of the 34 patients who had received brachytherapy, $17(15 \%)$ were treated with ${ }^{60} \mathrm{Co}$ HDR brachytherapy at a total dose of $20 \mathrm{~Gy}$ delivered in four fractions. In total, 17 (15\%) were treated with ${ }^{60} \mathrm{Co}$ HDR brachytherapy at a dose of $18 \mathrm{~Gy}$ delivered in six fractions. Between October 1, 2009 and June 30, 2012, electron beams (4, 6 and $9 \mathrm{MeV})$ were used, as they were more convenient for patients. The energy of the electron beams used was decided on the basis of the depth and location of the keloids. Radiotherapy schemes were 30 Gy delivered in 15 fractions $(30 \mathrm{~Gy} / 15 \mathrm{~F})$ or 26 Gy delivered in 13 fractions $(26 \mathrm{~Gy} / 13 \mathrm{~F})$.

The biologically effective dose (BED), as derived from the linear quadratic concept (5), was calculated for the various radiation regimens using the formula: $\mathrm{BED}=\mathrm{nd}[1+\mathrm{d} /(\alpha / \beta)]$, where $n$ is the number of fractions, $d$ is the dose per fraction, and $\alpha$ and $\beta$ are the parameters that determine the initial slope and degree of curvature of the underlying cell-survival curve, respectively. As keloids are considered to be acute-reacting tissues, it was assumed that $\alpha / \beta=10 \mathrm{~Gy}$ (6). According to this linear quadratic concept, the BED of each scheme was calculated, as shown in Table II.

Toxicity was graded according to the Common Terminology Criteria for Adverse Events [CTCAE v3.0 (http://ctep.info.nih. gov/CTC3/ctc.htm)]. An adverse effect $>90$ days after completion of radiotherapy was defined as a late adverse effect.

Surgical techniques. Local anesthesia was administered during almost all the surgical procedures. Excision outside the margin of the lesion was performed; the wound was closed sub-cutaneously with vicryl on which a plastic flexible tube was placed. The closed end of the tube was placed $>5 \mathrm{~mm}$ beyond the wound margin, and the open end extended $>10 \mathrm{~cm}$ beyond the wound margin to facilitate the connection of the tube to the HDR brachytherapy afterloader. The
Table I. Characteristics of the 116 patients.

\begin{tabular}{|c|c|}
\hline Characteristic & Patients, n $(\%$ \\
\hline \multicolumn{2}{|l|}{ Age, years } \\
\hline $10-20$ & $11(9.5)$ \\
\hline $21-30$ & $44(37.9)$ \\
\hline $31-40$ & $39(33.6)$ \\
\hline $41-50$ & $16(13.8)$ \\
\hline $51-60$ & $6(5.2)$ \\
\hline \multicolumn{2}{|l|}{ Gender } \\
\hline Female & $84(72.4)$ \\
\hline Male & $32(27.6)$ \\
\hline \multicolumn{2}{|l|}{ Localization of keloids } \\
\hline Sternum & $26(22.4)$ \\
\hline Ear lobe & $5(4.3)$ \\
\hline Ear auricle & $3(2.6)$ \\
\hline Haired occiput & $15(12.9)$ \\
\hline Back/scapulae & $24(20.7)$ \\
\hline Neck & $18(15.5)$ \\
\hline Shoulder & $21(18.1)$ \\
\hline Skin of chin & $1(0.9)$ \\
\hline Instep & $3(2.6)$ \\
\hline \multicolumn{2}{|c|}{ Dosage and type of radiation, dose/fractions } \\
\hline${ }^{192} \mathrm{Ir}$ HDR: $8 \mathrm{~Gy} / 1 \mathrm{~F}+9 \mathrm{~Gy} / 3 \mathrm{~F}$ & $22(19.0)$ \\
\hline${ }^{192}$ Ir HDR: $20 \mathrm{~Gy} / 4 \mathrm{~F}$ & $22(19.0)$ \\
\hline${ }^{60} \mathrm{Co}$ HDR: $20 \mathrm{~Gy} / 4 \mathrm{~F}$ & $17(14.7)$ \\
\hline${ }^{60} \mathrm{Co}$ HDR: $18 \mathrm{~Gy} / 6 \mathrm{~F}$ & $17(14.7)$ \\
\hline Electrons: $26 \mathrm{~Gy} / 13 \mathrm{~F}$ & $18(15.5)$ \\
\hline Electrons: $30 \mathrm{~Gy} / 15 \mathrm{~F}$ & $20(17.2)$ \\
\hline
\end{tabular}

BED, biologically effective dose; HDR, high dose rate; F, fractions; ${ }^{192} \mathrm{Ir}$, Iridium-192; ${ }^{60} \mathrm{Co}$, Cobalt-60.

Table II. Treatment scheme and BED of radiotherapy.

Dosage and type of radiation,

dosage/fractions

BED, Gy $(\alpha / \beta=10)$

\begin{tabular}{ll}
\hline${ }^{192} \mathrm{Ir}$ HDR: $8 \mathrm{~Gy} / 1 \mathrm{~F}+9 \mathrm{~Gy} / 3 \mathrm{~F}$ & 26.1 \\
${ }^{192} \mathrm{Ir}$ HDR: $20 \mathrm{~Gy} / 4 \mathrm{~F}$ & 30.0 \\
${ }^{60} \mathrm{Co}$ HDR: $20 \mathrm{~Gy} / 4 \mathrm{~F}$ & 30.0 \\
${ }^{60} \mathrm{Co}$ HDR: $18 \mathrm{~Gy} / 6 \mathrm{~F}$ & 23.4 \\
Electrons: $26 \mathrm{~Gy} / 13 \mathrm{~F}$ & 31.2 \\
Electrons: $30 \mathrm{~Gy} / 15 \mathrm{~F}$ & 36.0 \\
\hline
\end{tabular}

BED, biologically effective dose; HDR, high dose rate; F, fractions; ${ }^{192}$ Ir, Iridium-192; ${ }^{60} \mathrm{Co}$, Cobalt-60.

subcutaneous vicryl sutures act, among other effects, as a support for the tube. The wound was closed using a monocryl intracutaneous soluble suture. The scar, which is the junction of the two skin sections following excision, formed the target volume for the irradiation. The tube was placed as close as 
possible to the scar, such that the scar would be enclosed by a radius of $5 \mathrm{~mm}$ within the $100 \%$ isodose line. In case of wound dehiscence, two tubes were placed; the target volume was an oval enclosing the entire scar area. Subsequently, in the Department of Radiation Therapy, the irradiation was performed with an HDR afterloader using an Iridium-192 or Cobalt-60 source, which was slid into the tube for the irradiation. Upon administration of the last brachytherapy fraction, the tube was removed and the wound was covered with a gauze pad.

Statistical analysis. Patient data (patient characteristics and keloid characteristics) were collected using Excel. The recurrent time was defined as the days from the first day of radiotherapy to the date of recurrence. The patients who were known to remain with no recurrence at the time of the data update were censored at the dates of their last follow-up. The median follow-up time was calculated using the reverse Kaplan-Meier approach. The control rate was estimated using the Kaplan-Meier method, and the log-rank test was used to compare the differences between patient groups or sub-groups. The hazard ratio (HR) among groups was estimated using the proportional hazards regression with a 95\% Wald confidence interval (95\% CI). Data analysis was performed with SPSS version 19.0 (IBM Corp, Armonk, NY, USA), and all P-values were two-sided. $\mathrm{P}<0.05$ was considered to indicate a statistically significant difference.

\section{Results}

Patient follow-up. The median follow-up period was 46.5 months (range, 10-120 months) for all the patients. At the last observation date, 18 of the 116 patients had undergone relapse, and the median recurrent periods for the recurrent patients was 16.7 months (range, 10-30 months). The control rates for the different radiation regimens being compared are shown in Fig. 1. The median overall control rate for all the different radiotherapy techniques was $84.5 \%$. Although several differences could be identified from the curve of Fig. 1, no statistical difference occurred $(\mathrm{P}=0.094)$ for all the types of dosage and radiation.

Comparisons between subgroups. However, analyses comparing between the specific subgroups revealed certain differences. The control rate for the patients who received hypofractionated regimens $(>2 \mathrm{~Gy} / 1 \mathrm{~F})$ and conventional fractionation $(2 \mathrm{~Gy} / 1 \mathrm{~F})$ were 88.5 and $76.3 \%$, respectively ( $\mathrm{P}=0.043$; Fig. 2$)$.

The control rates for the patients who received a BED of $>30$ Gy and $<30$ Gy were 89.7 and $79.3 \%$, respectively ( $\mathrm{P}=0.104$; Fig. 3).

The control rates $(86.4$ vs. $95.5 \%)$ for the ${ }^{192} \mathrm{Ir}$ HDR (8 Gy/1F+9 Gy/3F) and ${ }^{192} \mathrm{Ir}$ HDR (20 Gy/4F) groups were not significantly different $(\mathrm{P}=0.286$; Fig. 4$)$. Of the 34 patients who received ${ }^{60} \mathrm{Co}$ HDR brachytherapy, a recurrence rate was observed in $11.7 \%$ of patients in the ${ }^{60} \mathrm{Co}$ HDR $20 \mathrm{~Gy} / 4 \mathrm{~F}$ group and $17.6 \%$ of patients in the ${ }^{60} \mathrm{Co}$ HDR $18 \mathrm{~Gy} / 6 \mathrm{~F}$ group $(\mathrm{P}=0.622$; Fig. 5). In the electron beam irradiation groups, the control rates for 66.7 vs. $85.0 \%$ of the electron $26 \mathrm{~Gy} / 13 \mathrm{~F}$ and $30 \mathrm{~Gy} / 15 \mathrm{~F}$ groups, respectively, were not significantly different $(\mathrm{P}=0.141$; Fig. 6).
There were no grade 2 or higher adverse effects based on the CTCAE v3.0 in the late phase. Of all the 116 patients studied, two patients were diagnosed with middle esophageal cancer, one 5 years after treatment and another 6 years after. The locations of the keloids were the neck, and the irradiated areas were a distance away from the middle esophagus. Therefore, whether there is any association between radiotherapy and cancer cannot be confirmed, due to the high incidence rate of esophageal cancer in northern China.

\section{Discussion}

Keloids have been treated using radiation for more than a century, and the value of radiotherapy in the treatment of keloids has been established for almost a century. The mechanism by which radiation prevents regrowth of keloids is unknown. A previous study showed that fibroblasts were destroyed by radiation and were not replaced by blood-borne cells from distant tissues (7). By destroying a sufficient number of cells, a balance could be created between collagen synthesis and degradation.

In the present study, the recurrence rates of keloids following postoperative radiation therapy using HDR Iridium-192, HDR Cobalt-60 and electron beams were analyzed. As mentioned previously, the shapes of the curves suggest differences in the various groups, but no significant differences were observed when the curves were analyzed and compared using log-rank tests. However, compared to the three different techniques, the pooled data for all 116 patients showed that the control rate for patients who received hypofractionated $(>2 \mathrm{~Gy} / 1 \mathrm{~F})$ radiotherapy was significantly higher than that for the patients who received conventional fractionated $(2 \mathrm{~Gy} / 1 \mathrm{~F})$ radiotherapy.

This result indicated that hypofractionated radiotherapy played an important role in the adjuvant therapy following surgical excision of keloids. Flickinger et al (8) used a comprehensive literature review and a database of 2,515 resected keloids to identify factors that significantly affected recurrence rates following postoperative external beam radiotherapy of keloids, and to delineate any radiation dose response and effects of radiation dose per fraction. The results showed that the relatively low $\alpha / \beta$ ratio indicates that radiotherapy with a limited number of fractions and high doses per fraction is the optimum strategy. The present results support this conclusion.

It is widely accepted that the $\alpha / \beta$ ratio is equal to $\sim 10 \mathrm{~Gy}$ for acute reacting tissues, in the range of 1 to $3 \mathrm{~Gy}$ for late-reacting tissues. The study by Kal and Veen (6) assumed keloids to be one of these acute-reacting tissues, and applied an $\alpha / \beta$ rate of $10 \mathrm{~Gy}$. However, the review by Flickinger et al (8) showed that the radiobiological dose response function for postoperative radiotherapy of keloids has a low $\alpha / \beta$ ratio, with a mean value of 2.08 from the different models (and maximum 95\% confidence values of 3.72-5.40). The differences in $\alpha / \beta$ values were mainly between those from the model database and the higher values obtained in the expanded database. The low overall average $\alpha / \beta$ ratio value of 2.08 identified for postoperative keloid radiotherapy was comparable to values of 1.9 to 3.1 for late fibrosis and 2.8 to 3.7 for telangiectasia following breast radiotherapy $(5,9,10)$. The low $\alpha / \beta$ ratio for postoperative keloid radiotherapy and the relatively high BED 


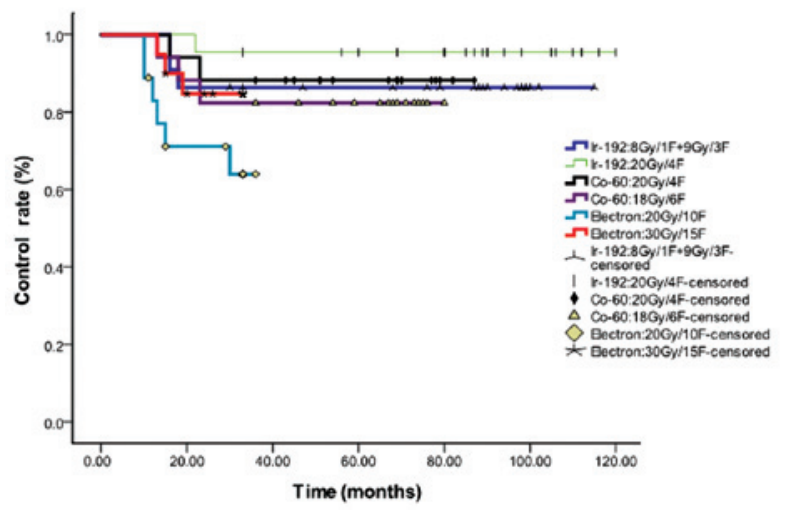

Figure 1. Overall control rate for the different radiotherapy techniques. Ir, iridium; Co, cobalt.

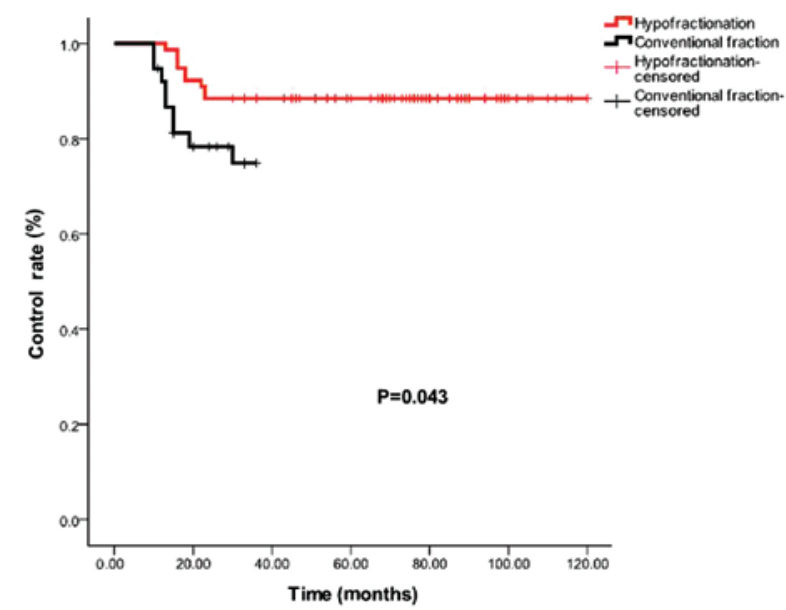

Figure 2. Control rates for hypofractionated (>200 cGy per fraction) (red) and conventional fraction (200 cGy per fraction) (black).

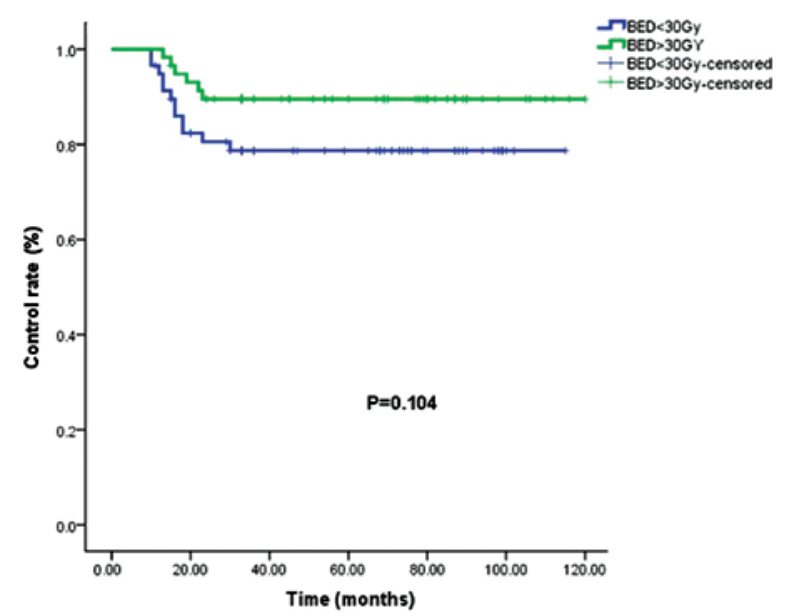

Figure 3. Control rate for BED $>30$ Gy (green) and $<30$ Gy (blue). BED, biologically effective dose.

time/repopulation correction factor indicate that there is no advantage of increased fractionation in this treatment.

Surgical resection followed by radiotherapy, despite a variety of doses administered, produced an acceptably low

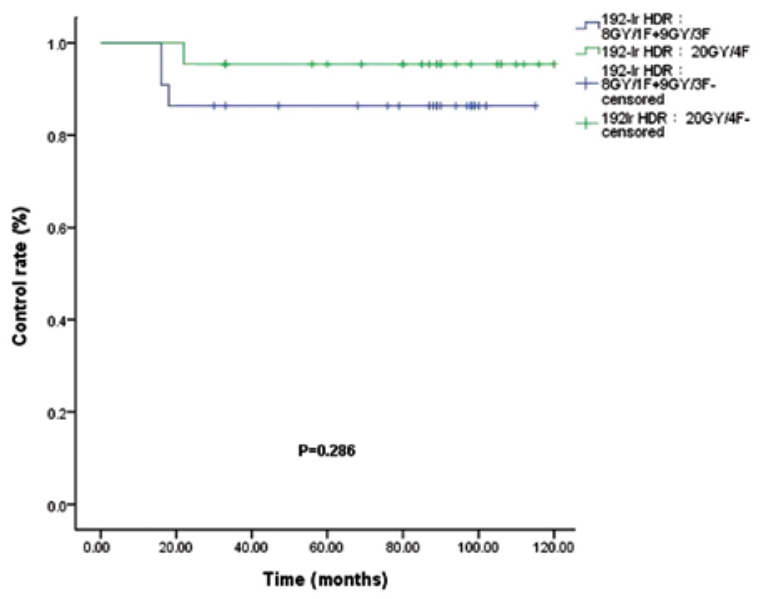

Figure 4. Control rates for ${ }^{192} \mathrm{Ir}$ HDR ( $\left.8 \mathrm{~Gy} / 1 \mathrm{~F}+9 \mathrm{~Gy} / 3 \mathrm{~F}\right)$ (blue) and ${ }^{192} \mathrm{Ir}$ HDR (20 Gy/4F) (green). Ir, ididium; HDR, high-dose rate.

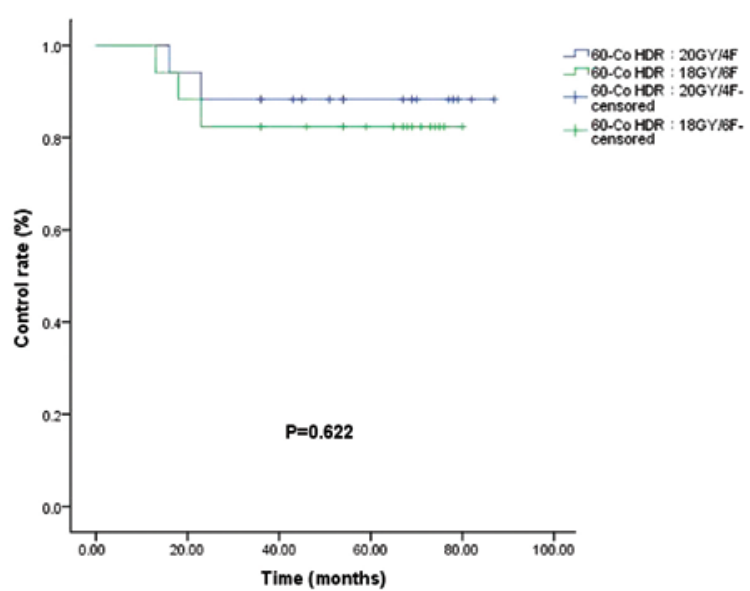

Figure 5. Control rates for ${ }^{60} \mathrm{Co}$ HDR $18 \mathrm{~Gy} / 6 \mathrm{~F}$ (green) and $20 \mathrm{~Gy} / 4 \mathrm{~F}$ (blue). Co, cobult; HDR, high-dose rate.

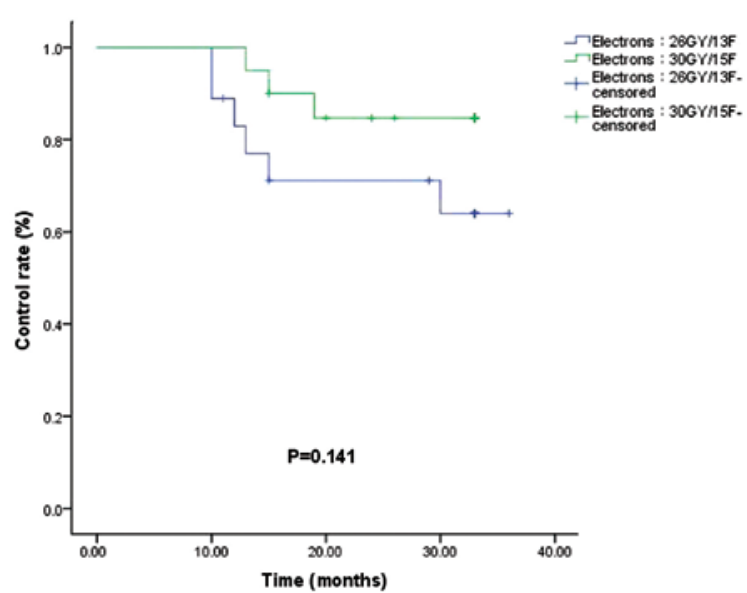

Figure 6. Control rates for electrons at $26 \mathrm{~Gy} / 13 \mathrm{~F}$ (blue) and $30 \mathrm{~Gy} / 15 \mathrm{~F}$ (green).

recurrence rate. However, no consensus has been reached on the total dosage, fractionation or optimal timing of the delivery of radiotherapy. The present study analyzed the recurrence rates of keloids following postoperative radiation therapy using 
the linear quadratic-BED concept with the aim of normalizing the radiotherapy doses into BEDs to be able to construct dose-effect associations. The study by Kal and Veen (6) reviewed the literature for the recurrence rates of keloids subsequent to surgical excision followed by radiotherapy. In total, 18 studies were identified that provided data on the recurrence rate and administered dose. Their results indicated a dose-effect association for the recurrence rate of keloids as a function of the BED, and they suggested that a BED of $>30$ Gy must be applied within 1-2 days after surgery. The present data (Fig. 3) were consistent with the conclusions of Kal and Veen (6), but the differences between the $<30$ Gy and $>30$ Gy groups in the present study were significantly different in the analysis. Assessing whether this lack of significance reflects the limited sample size in the present study, or whether other variable factors, such as target volume, dose/fraction depth of radiotherapy, surgical approach and location of the keloid, also contributed is difficult.

The risk of a fatal tumor is of particular concern to the majority of patients; plastic surgeons tend to avoid radiation therapy for keloids for fear of inducing malignant tumors. The International Commission on Radiological Protection provided risk factors for a number of organs, but not for fat or muscle tissues (11). Ogawa et al (12) searched for previous studies of carcinogenesis associated with radiation therapy for keloids and examined the evidence-based opinions of radiation oncologists regarding the acceptability of radiation therapy for the treatment of keloids. This study noted that the volume irradiated during the treatment of keloids is extremely small, and as a consequence of the small volume irradiated, the risk of a radiation-induced tumor is negligible. The study concluded that the risk of carcinogenesis from keloid radiation therapy was extremely low when performed with appropriate doses and under conditions that provide adequate protection of surrounding tissues, including the thyroid and the breast, particularly in children and infants, and that radiation therapy was acceptable as a keloid treatment modality. For the treatment of keloids in the present study, as a result of the use of HDR brachytherapy and electron beams with a rapid fall-off of the dose, the treated volume was extremely small and the probability of tumor induction would also be negligible, but further observations are required.

In conclusion, the results of the present study indicate that hypofractionated radiotherapy can play an important role in the adjuvant therapy following surgical excision of keloids. A BED of $>30$ Gy appears to be sufficient. No definitive evidence was found between radiotherapy and the occurrence of cancer during the follow-up, but more cases and longer follow-up periods are required.

\section{Acknowledgements}

The authors would like to thank Dr Sara Rockwell (Department of Therapeutic Radiology, Yale University School of Medicine, New Haven, CT, USA) for her modifications to the language, comments and suggestions during the preparation of the manuscript. The present study was supported by the Key Academic Discipline Projects, Hubei University of Medicine (grant no. 2014XKJSXJ12 to Dr Zhiguo Luo).

\section{References}

1. Rubin P, Soni A and Williams JP: The molecular and cellular biologic basis for the radiation treatment of benign proliferative diseases. Semin Radiat Oncol 9: 203-214, 1999.

2. Ramakrishnan KM, Thomas KP and Sundararajan CR: Study of 1,000 patients with keloids in South India. Plast Reconstr Surg 53: 276-280, 1974.

3. Sakamoto T, Oya N, Nagata Y and Hiraoka M: The outcome and the adverse effect of postoperative radiotherapy for keloids: A prospective study with a total dose of $20 \mathrm{GY}$. Int J Radiat Oncol Biol Phys 60 Suppl 1: 549-550, 2004.

4. Leventhal D, Furr M and Reiter D: Treatment of keloids and hypertrophic scars: a meta-analysis and review of the literature. Arch Facial Plast Surg 8: 362-368, 2006.

5. Barendsen GW: Dose fractionation, dose rate and iso-effect relationships for normal tissue responses. Int J Radiat Oncol Biol Phys 8: 1081-1997, 1982.

6. Kal HB and Veen RE: Biologically effective doses of postoperative radiotherapy in the prevention of keloids. Dose-effect relationship. Strahlenther Onkol 181: 717-723, 2005.

7. Ragoowansi R, Cornes PG, Glees JP, Powell BW and Moss AL: Ear-lobe keloids: treatment by a protocol of surgical excision and immediate postoperative adjuvant radiotherapy. Br J Plast Surg 54: 504-508, 2011.

8. Flickinger JC: A radiobiological analysis of multicenter data for postoperative keloid radiotherapy. Int J Radiat Oncol Biol Phys 79: 1164-1170, 2011.

9. Baltas D, Fehrentz D and Turesson I: Analysis of late effects data using dose-response models: application to human skin telangiectasia data. Radiother Oncol 16: 41-53, 1989.

10. Chevray PM and Manson PN: Keloid scars are formed by polyclonal fibroblasts. Ann Plast Surg 52: 605-608, 2004.

11. ICRP. International commission on radiological protection. 1990 recommendations of the international commission on radiological protection. ICRP Publication 60. Ann ICRP 21: 1-3, 1991.

12. Ogawa R, Yoshitatsu S, Yoshida K and Miyashita T: Is radiation therapy for keloids acceptable? The risk of radiation-induced carcinogenesis. Plast Reconstr Surg 124: 1196-1201, 2009. 